\title{
Modelo de localização ótima de estações ferroviárias: a futura linha de alta velocidade Lisboa-Porto
}

\author{
Hugo M. Repolho ${ }^{1}$, António P. Antunes ${ }^{2}$ e Richard L. Church ${ }^{3}$
}

\begin{abstract}
Resumo: Nas últimas décadas assistiu-se ao renascimento do transporte ferroviário, estando previstos investimentos avultados nos próximos anos, especialmente no que respeita à alta velocidade. O sucesso deste tipo de investimentos depende fortemente do volume de passageiros captados, o qual depende por sua vez do número e localização das estações ferroviárias. Este artigo apresenta um modelo de otimização inteiro-misto para determinar o número e localização ótimos das estações intermédias de uma linha de trens de alta velocidade. As estações são escolhidas de entre um conjunto de locais possíveis definidos a priori com o objetivo de maximizar a diminuição dos custos de viagens. O modelo tem em conta a sensibilidade da demanda ao tempo de viagem perdido nas paragens intermédias, bem como a competição com modos de transporte alternativos. A utilidade prática do modelo é ilustrada através de uma aplicação à futura linha de alta velocidade Lisboa-Porto. DOI:10.4237/transportes.v20i3.555.
\end{abstract}

Palavras-chave: trem de alta-velocidade, localização de estações, demanda elástica.

\begin{abstract}
Rail transportation reflourished in the last few decades. Large investment in new railway lines is expected to be made in the years to come - especially in high speed rail lines. The success of such investment is highly dependent on rail ridership, which in turn depends on the location of railway stations. This paper proposes a mixed-integer optimization model that determines the optimal location (and number) of stations along a railway line that will be introduced over an existing transportation network. The stations are chosen within a set of possible locations defined a priori according to the objective of maximizing travel cost savings. The model considers the sensitivity of rail ridership to time losses due to stops at intermediate stations, as well as competition from other modes. The usefulness of the model in real-world situations is illustrated with a case study involving a high speed rail line expected to be built in Portugal in the near future: the Lisbon-Porto line.
\end{abstract}

Keywords: high-speed-rail, station-location, elastic demand.

\section{INTRODUÇÃO}

Após um período de estagnação e até mesmo de retrocesso, o transporte ferroviário renasceu nas últimas décadas, particularmente devido a um interesse crescente nas linhas de alta velocidade. Os primeiros projetos de alta velocidade surgiram no Japão e na Europa. Na Europa, eles foram inicialmente desenvolvidos à escala nacional em França, Alemanha e Itália, tendo depois evoluído para a escala europeia. Com efeito, desde 1996 os projetos de alta velocidade têm sido impulsionados no âmbito do Programa da Rede Trans-Europeia de Transportes (European Union, 1996, 2001; Nash, 2009). O investimento em projetos de alta velocidade é hoje uma tendência crescente também na Ásia e na América do Norte e do Sul, incluindo no Brasil, onde está planeada a construção de uma linha entre São Paulo e o Rio de Janeiro (ANTT, 2011).

O transporte ferroviário é hoje apontado como uma solução para os problemas de congestionamento que atualmente se verificam nas redes rodoviárias e nos aeroportos (Vuchic e Casello, 2002). Para além disso, o transporte ferroviário apresenta-se como uma alternativa ambientalmente mais sustentável (menor consumo de energia e de emissões de gases causadores do efeito de estufa) e com um índice de

\footnotetext{
${ }^{1}$ Hugo M. Repolho, Departamento de Engenharia Civil, Universidade de Coimbra, Coimbra, Portugal. (e-mail: repolho@dec.uc.pt).

${ }^{2}$ António P. Antunes, Departamento de Engenharia Civil, Universidade de Coimbra, Coimbra, Portugal. (e-mail: antunes@dec.uc.pt).

${ }^{3}$ Richard L. Church, Department of Geography, University of California Santa Barbara, Santa Barbara, California, EUA. (e-mail: church@geog.ucsb.edu).

Manuscrito recebido em 28/2/2012 e aprovado para publicação em 14/11/2012. Este artigo é parte de TRANSPORTES v. 20, n. 3, 2012. ISSN: 2237-1346 (online). DOI:10.4237/transportes.v20i3.555.
}

sinistralidade muito baixo. A alta velocidade é considerada, a par com o transporte aéreo, um dos meios de transporte mais seguros (Campos e De Rus, 2009). No contexto europeu há ainda uma outra razão que favorece a alta velocidade: a necessidade de reduzir o tempo médio de viagem entre capitais europeias, a qual aumentou significativamente depois da inclusão de diversos países de Leste.

O desenvolvimento de uma rede de alta velocidade é um processo extremamente complexo e dispendioso. O sucesso dos investimentos depende em grande medida do número de passageiros servidos (De Rus e Nombela, 2007; Campos e De Rus, 2009), em particular dos captados a outros meios de transporte. A atratividade das linhas de alta velocidade pode ser medida em função da diminuição dos custos de viagem em relação aos meios de transporte existentes, bem como da quantidade de demanda gerada. A diminuição dos custos de viagem depende do número e localização das estações - mais estações implicam menor tempo de acesso aos serviços ferroviários e, consequentemente, a atração de mais demanda. Contudo, implicam também um maior número de paragens intermédias para as viagens mais longas e, consequentemente, tempos de viagem mais longos devido às fases de travagem, desembarque, embarque e aceleração adicionais. $\mathrm{O}$ aumento dos tempos de viagem provoca a diminuição da demanda para viagens de longo curso. Em resumo, cada estação adicional aumenta a demanda local mas tende a diminuir a demanda global.

Este artigo apresenta um modelo de planeamento estratégico de investimentos ferroviários (Ghoseiri et al., 2004). Trata-se de um modelo de otimização inteiro-misto que determina o número e localização ótimos de estações ao longo de uma linha ferroviária que vai ser construída sobre uma rede de transportes já existente. As estações são escolhidas de entre um conjunto de locais possíveis definidos a priori 
com o objetivo de maximizar a diminuição dos custos de viagem do sistema. O modelo considera o efeito na demanda captada das perdas de tempo devido a paragens em estações intermédias, e a competição com modos de transporte alternativos. O traçado do corredor ferroviário é um dado do problema.

$\mathrm{O}$ artigo encontra-se organizado da seguinte forma. A próxima secção apresenta uma breve revisão da literatura sobre modelos de otimização de localização de estações. De seguida é formulado o novo modelo de localização de estações e aplicado a um estudo de caso envolvendo a futura linha de alta velocidade Lisboa e Porto, em Portugal. Descrito o estudo de caso, os resultados obtidos são analisados e comparados com as soluções consideradas em estudos existentes sobre a nova linha. $\mathrm{O}$ artigo termina com uma síntese dos trabalhos efetuados e com a identificação de possíveis desenvolvimentos futuros.

\section{REVISÃO DE LITERATURA}

O planeamento estratégico de investimentos ferroviários aborda dois problemas fundamentais profundamente interligados - a escolha do corredor e a escolha da localização das estações - tendo ambos sido tema de diversos artigos científicos, particularmente no contexto das redes de transporte urbano rápido (rapid transit networks). Laporte et al. (2000) contém uma revisão da literatura existente sobre o tema. Ainda que similares em vários aspetos, as redes de transportes urbanos rápidos e as redes ferroviárias variam no ambiente de aplicação. Enquanto as primeiras se desenvolvem à escala da cidade ou da área metropolitana e são frequentemente bastante densas, as segundas dizem respeito a espaços interurbanos e são normalmente pouco densas (no caso das linhas de alta velocidade pode mesmo tratar-se apenas de uma linha). A revisão bibliográfica que se segue centra-se apenas em estudos sobre modelos de localização de estações nos quais o traçado do corredor é um dado do problema.

O primeiro artigo relevante sobre modelos de localização de estações foi apresentado por Vuchic e Newell (1968). O modelo formulado por estes autores tem em conta todos os ingredientes de um problema de localização de estações velocidade de acesso às estações, características dinâmicas dos trens (fase de aceleração, fase de velocidade constante e fase de travagem), tempo de viagem dentro do trem e tempo de transferência intermodal nas estações - mas destina-se ao caso específico de viagens entre a periferia e a área central de uma cidade. A solução do modelo é obtida através de um sistema de equações diferenciais cuja resolução permite determinar o espaçamento entre estações que minimiza o tempo total de viagens. A demanda é expressa por uma função contínua da distância ao ponto central da cidade. Um modelo similar foi proposto em Vuchic (1969), com uma função objetivo diferente e considerando a competição de um sistema de transportes contínuo (acessível em qualquer ponto) e paralelo à linha ferroviária. O objetivo do novo modelo é a maximização do número de utilizadores da linha ferroviária, admitindo que as viagens são feitas pelo caminho mais rápido.

O problema da localização de estações foi novamente abordado por Laporte et al. (1998) através da formulação de um modelo que procura localizar um número fixo de estações ao longo de uma linha ferroviária, de forma a maxi- mizar a demanda captada, a qual é estimada em função da área coberta pelas estações. O modelo não tem em conta o impacto do aumento do tempo de viagem devido a paragens em estações intermédias. A utilidade do modelo foi ilustrada através da aplicação a uma linha de transportes urbanos rápidos de Sevilha (Espanha).

Outro contributo significativo para o problema de localização de estações foi dado por Hamacher et al. (2001). Os autores deste artigo introduziram dois modelos de localização contínua de estações: o primeiro com o objetivo de maximizar a acessibilidade às estações e o segundo com o objetivo de maximizar a diminuição do tempo total de viagens. O segundo modelo considera os efeitos positivos e negativos de localizar estações adicionais numa linha ferroviária já existente. $\mathrm{O}$ acréscimo de tempo de viagem é tido em conta para calcular o tempo total de viagem, mas a sua repercussão na demanda é ignorada. Os modelos foram testados para um estudo de caso envolvendo a Alemanha. No mesmo âmbito, Schöbel et al. (2002) apresenta um modelo discreto de cobertura para o problema da minimização do número (custo) de estações adicionais, ao mesmo tempo que garantiam a cobertura de toda a demanda da rede. Por sua vez, Kranakis et al. (2003) descreve um modelo de cobertura máxima para um número fixo de estações. Apesar do artigo mencionado discutir a necessidade de incluir custos adicionais devido à paragem em estações intermédias, esse aspeto não foi contemplado no modelo final. Schöbel (2005) estende o modelo apresentado em Schöbel et al. (2002) através da formulação de um modelo com dois objetivos: maximizar a demanda coberta e minimizar o número de estações. Finalmente, a abordagem proposta em Shöbel (2005) foi generalizada em Gross et al. (2009), através da consideração de duas funções objetivo distintas: minimização do número de paragens garantindo que todos os centros geradores de tráfego são servidos por uma estação localizada a uma distância inferior ao raio de cobertura mínimo definido; e minimização da soma das distâncias entre centros geradores de tráfego e as estações mais próximas dado um número fixo de novas estações.

Em suma, existem na literatura vários modelos de otimização para o problema da localização de estações. A grande maioria dos modelos utiliza a demanda na área coberta pelas estações como indicador do volume de passageiros captados. No entanto, e conforme referido por Marín e Jaramillo (2009), o facto de uma rede de transportes cobrir a demanda não significa que ela responda adequadamente ao padrão de viagens dos utilizadores. Alguns modelos focam a questão da diminuição dos custos/tempo de viagem tornando possível a modelação do comportamento dos utilizadores de forma mais precisa. No entanto, não temos conhecimento de nenhum modelo que capte todas as particularidades inerentes ao problema da localização de estações (em particular das implicações do número de paragens intermédias na demanda captada). Na literatura existente, os modelos que abordam o problema em estudo de forma mais abrangente são os modelos originais formulados por Vuchic e Newell (1968) e Vuchic (1969), apesar de se aplicarem somente ao caso específico de uma rede em que toda a demanda tem um único destino (a área central de uma cidade). 


\section{MODELO DE OTIMIZAÇÃO}

O modelo de localização de estações apresentado neste artigo pode ser visto como uma generalização dos modelos de Vuchic e Newell (1968) e Vuchic (1969). O modelo considera todas as particularidades abordadas nesses trabalhos, mas generaliza a sua aplicação a qualquer nova linha ferroviária, assumindo que a demanda está localizada num conjunto finito de centros geradores de tráfego, isto é os locais onde existem potenciais utilizadores da linha ferroviária ou os locais que são polos de destino dos utilizadores. $\mathrm{O}$ modelo aqui apresentado considera que os polos de origem e destino são coincidentes e serão numa escala macro correspondentes às cidades existentes na região atravessada pela nova linha ferroviária. O objetivo é determinar a localização de estações ao longo de um corredor ferroviário previamente definido, de forma a maximizar a diminuição dos custos de viagem decorrente da introdução da nova linha ferroviária. O conjunto de locais possíveis para as estações é definido a priori. O novo serviço ferroviário compete com os modos alternativos que operam na rede de transportes existente. A demanda é calculada com base numa matriz origem-destino entre os centros geradores de tráfego tendo em conta os custos de viagem. Esta abordagem, tal como referido em Laporte et al. (2000), é mais realista do que estimar a demanda com base no raio de cobertura das estações. Os custos de viagem incluem o tempo perdido devido a possíveis paragens em estações intermédias.

O modelo aplica-se a uma região onde se pretende construir uma nova linha ferroviária. Os dados referentes aos conjuntos de centros geradores de tráfego e das localizações potenciais de estações ferroviárias são definidos da seguinte forma:

- $\boldsymbol{J}$ : Conjunto de centros geradores de tráfego;

- $\boldsymbol{M}=\{1, \ldots, M\}$ : Conjunto de localizações possíveis para as estações;

- $\boldsymbol{R}_{m n}$ : Conjunto das possíveis estações intermédias entre as estações $m$ e $n$;

- $r_{m n}$ : Número máximo de estações intermédias possíveis entre as estações $m$ e $n$; e

- $d_{m n}$ : Distância entre as estações $m$ e $n$ pela nova linha ferroviária.

Um dos principais tipos de dados do modelo diz respeito aos custos de viagem pela rede de transportes existente e pela nova linha ferroviária. Os custos de viagem dependem dos tempos de viagem (incluindo o tempo perdido nas trocas de modo) e dos custos inerentes a cada tipo de transporte. Os parâmetros necessários para caracterizar os custos de viagem são definidos da seguinte forma:

- $\quad c_{i j}$ : Custo de viagem entre os centros $i$ e $j$ através da rede de transportes existente;

- $c_{i m}\left(c_{n j}\right)$ : Custo de viagem entre o centro $i(j)$ e a estação $m(n)$ através da rede de transportes existente;

- $\quad c_{m n}$ : Custo de viagem entre as estações $m$ e $n$ através da nova linha ferroviária quando o trem se desloca à velocidade máxima (o custo é composto pelo preço do bilhete e o custo do tempo de viagem, que inclui a fase de aceleração na estação de origem, $m$, e a fase de desaceleração junto da estação de destino, $n$ );

- $v$ : Custo unitário do tempo;

- $t^{e}$ : Tempo perdido na mudança de modos de transporte (trem-carro);
- $t^{s}$ : Tempo perdido em cada paragem intermédia (inclui embarque e desembarque, e as fases de desaceleração e aceleração);

- $c_{i m n j}^{k}=c_{i m}+c_{m n}+c_{n j}+2 v t^{e}+k v t^{s}$ : Custo de viagem entre os centros $i$ e $j$ para uma rota que inclui dois segmentos da rede de transportes existente, im e $j n$, e um segmento da nova linha ferroviária $m n$, que contém $k$ paragens intermédias;

- $s_{i m n j}^{k}=c_{i j}-c^{k}{ }_{i m n j}$ : Valor da diminuição dos custos viagens entre os centros $i$ e $j$ devido à utilização de um segmento da nova linha ferroviária $m n$;

- $g_{m}^{a}\left(g_{n}^{e}\right)$ : Limite superior para o número de rotas que utilizam a estação $m(n)$ como acesso à (saída da) linha ferroviária; e

- $l_{\text {min }}$ : Distância mínima entre duas estações consecutivas.

Um outro tipo de dados necessário diz respeito ao número de viagens que se tornam mais económicas se um segmento da nova linha ferroviária for utilizado. Este número é representado por $q^{k}{ }_{i m n j}$ para a rota $i \rightarrow m \rightarrow n \rightarrow j$ com $k$ paragens intermédias, e pode ser estimado em função do custo de viagem da rota $\left(c^{k}{ }_{i m n j}\right)$ usando um modelo gravitacional (Ortúzar and Willumsen, 2001). Se o custo dessas viagens for menor que o custo das viagens feitas somente através da rede de transportes existente $\left(c^{k}{ }_{i m n j}<c_{i j}\right)$, o valor de $q^{k}{ }_{i m n j}$ capta ainda as viagens adicionais geradas pela diminuição do custo de viagem. Caso contrário $q^{k}{ }_{i m n j}$ toma o valor zero. Daqui se depreende que se assume que os potenciais utilizadores só escolhem a nova linha ferrovária caso esta tenha um custo de viagem inferior ao da rota pela rede de transportes existente correspondente.

As variáveis de decisão são a localização das estações, $y_{m}$, e as rotas escolhidas pelos utilizadores, $x_{i m n j}^{k}$, ambas variáveis binárias: as variáveis $y_{m}$ tomam o valor 1 se uma estação $m$ for seleccionada e 0 em caso contrário; e as variáveis $x_{i m n j}^{k}$ tomam o valor 1 se a rota $i \rightarrow m \rightarrow n \rightarrow j$ com $k$ paragens intermédias for escolhida e 0 em caso contrário. $\mathrm{O}$ modelo utiliza matrizes de viagens $\mathrm{O} / \mathrm{D}$ e de custos de viagem simétricas pelo que é possível otimizar apenas um sentido $(i<j)$ sem perda de generalização.

Por fim, é necessário definiu-se ainda uma distância mínima entre duas estações consecutivas, $l_{\min }$, por forma a garantir o carácter interregional do serviço ferroviário, e ainda um limite máximo e mínimo para o número de rotas que utilizam uma estação como ponto de acesso ou saída da linha ferroviária, $g_{m}^{a}$ e $g_{n}^{e}$. Notar que estes limites podiam ser definidos como um número extremamente grande (Big $M$ ), no entanto por motivos de eficiência computacional é preferível calcular com exactidão qual poderão ser esses limites máximos. Como tal estação é calculado a priori o número de rotas que poderão potencialmente usar uma estação $f(g)$ como ponto de acesso (saída), o qual é igual ao número de parâmetros $q^{k}{ }_{i f n j}\left(q^{k}{ }_{i m g j}\right)$ diferentes de zero.

Nestas condições, a localização das estações que maximiza a diminuição dos custos de viagem da rede pode ser determinada através do seguinte modelo de otimização:

$$
\text { Maximizar } 2 \sum_{i \in \boldsymbol{J}} \sum_{m \in \boldsymbol{M}} \sum_{n \in \boldsymbol{M}} \sum_{j \in \boldsymbol{J}} \sum_{k=0}^{r_{m n}} s_{i m n j}^{k} q_{i m n j}^{k} x_{i m n j}^{k}
$$




$$
\begin{aligned}
& \sum_{m \in M} \sum_{n \in M} \sum_{k=0}^{r_{m n}} x_{i m n j}^{k} \leq 1 \quad \forall i, j \in \boldsymbol{J} \\
& \sum_{i \in \boldsymbol{J}} \sum_{n \in \boldsymbol{M}} \sum_{j \in \boldsymbol{J}} \sum_{k=0}^{r_{m n}} x_{i m n j}^{k} \leq g_{m}^{a} y_{m} \quad \forall m \in \boldsymbol{M}: g_{m}^{a}>0 \\
& \sum_{i \in \boldsymbol{J}} \sum_{m \in \boldsymbol{M}} \sum_{j \in \boldsymbol{J}} \sum_{k=0}^{r_{m n}} x_{i m n j}^{k} \leq g_{n}^{e} y_{n} \quad \forall n \in \boldsymbol{M}: g_{n}^{e}>0 \\
& y_{m}+y_{m+1} \leq 1 \quad \forall m \in \boldsymbol{M}: d_{m, m+1} \leq l_{\text {min }} \\
& \sum_{k=0}^{r_{m n}}\left(r_{m n}-k\right) x_{i m n j}^{k} \leq r_{m n}-\sum_{u \in \boldsymbol{R}_{m n}} y_{u} \\
& \forall i, j \in \boldsymbol{J}, m, n \in \boldsymbol{M}: x_{i m n j}^{0} \text { exists } \\
& y_{1}=1 \\
& y_{M}=1 \\
& x_{i m n j}^{k} \in\{0,1\} \quad \forall i, j \in \boldsymbol{J}, m, n \in \boldsymbol{M} \\
& y_{m} \in\{0,1\} \quad \forall m \in M
\end{aligned}
$$

A função objetivo (1) maximiza a diminuição dos custos totais de viagem, $s^{k}{ }_{i m n j}$, da rede devido à introdução da linha ferroviária (a multiplicação por dois é feita para se considerarem os dois sentidos de viagem). As restrições de atribuição (2) asseguram que as viagens entre cada par O/D são atribuídas no máximo a uma rota incluindo um segmento $(m n)$ da nova linha ferroviária. Nesse caso, $x^{k}{ }_{i m n j}=1$ se exis- tirem $k$ estações intermédias entre $m$ e $n$. Se as viagens forem feitas somente pela rede de transportes existente então $x^{k}{ }_{i m n j}=0$, para qualquer valor de $k$. As restrições (3) evitam que sejam atribuídas viagens a rotas com o segmento ferroviário $m n$ se não existirem estações em $m$ e $n$. Neste caso qualquer $x_{i m n j}^{k}$ toma valor igual a um se forem localizadas estações em $m\left(y_{m}=1\right)$ e $n\left(y_{n}=1\right)$ e zero caso contrário. As restrições (4) garantem que a distância entre duas estações consecutivas, $y_{m}$ e $y_{m+1}$, é igual ou superior à distância mínima $l_{\text {min }}$ definida pelo decisor. As restrições (5) determinam o número de estações intermédias localizadas entre duas estações (como é prática corrente nas fases de planeamento estratégico assume-se que os trens param em todas as estações existentes). Estas restrições só são formuladas se $x^{0}{ }_{i m n j}$ existir, isto é, se pelo menos a rota que inclui um segmento da nova linha ferroviária sem estações intermédias $(k=0)$ tiver um custo de viagem inferior à melhor rota alternativa que utiliza apenas a rede de transportes existente. As restrições (6a) e (6b) localizam uma estação em cada extremidade da nova linha ferroviária. Finalmente, as expressões (7) e (8) definem as variáveis de decisão como variáveis binárias.

\section{APLICAÇÃO PRÁTICA}

A utilidade do modelo de localização de estações proposto é ilustrada através de uma aplicação à futura linha de alta velocidade Lisboa-Porto. Esta linha faz parte do projecto prioritário número 3 (entre 30) definido pela Comissão Europeia, sendo uma componente importante do eixo de alta velocidade do Sudoeste da Europa A linha atravessa as áreas mais populosas e economicamente desenvolvidas de Portugal, as quais são servidas por uma rede rodoviária moderna que competirá com a linha de alta velocidade. Para além da linha de alta velocidade existe ainda o serviço ferroviário convencional e um serviço de transporte aéreo
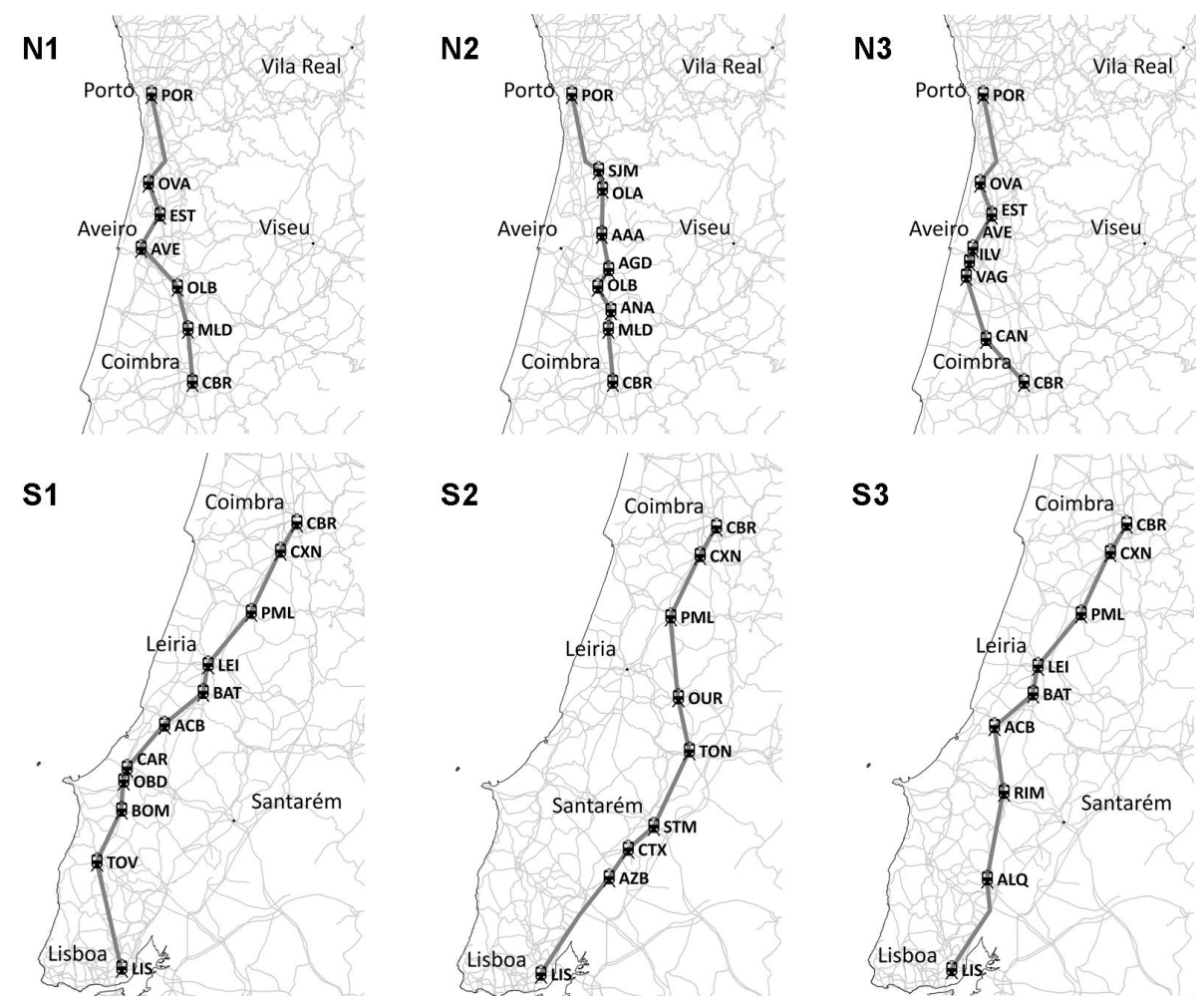

Figura 1. Corredores alternativos a norte e sul de Coimbra 
Tabela 1. Munícipos candidatos à localização de estações

\begin{tabular}{ll|ll|ll}
\hline Localização & Abv. & Localização & Abv. & Localização & Abv. \\
\hline Águeda & AGD & Cartaxo & CTX & Ourém & OUR \\
Albergaria-a-Velha & AAA & Coimbra & CBR & Ovar & OVA \\
Alcobaça & ACB & Condeixa-a-Nova & CXN & Pombal & PML \\
Alenquer & ALQ & Estarreja & EST & Porto & POR \\
Anadia & ANA & Ílhavo & ILV & Rio Maior & RIM \\
Aveiro & AVE & Leiria & LEI & Santarém & STM \\
Azambuja & AZB & Lisboa & LIS & São João da Made & SJM \\
Batalha & BAT & Mealhada & MLD & Torres Novas & TON \\
Bombarral & BOM & Óbidos & OBD & Torres Vedras & TOV \\
Caldas da Rainha & CAR & Oliveira de Azeméis & OLA & Vagos & VAG \\
Cantanhede & CAN & Oliveira de Barro & OLB & & \\
\hline
\end{tabular}

Tabela 2. Traçado, extensão e número máximo de estações intermédias dos corredores

\begin{tabular}{llllllllll}
\hline Corredor & COR1 & COR2 & COR3 & COR4 & COR5 & COR6 & COR7 & COR8 & COR9 \\
\hline Sul & S1 & S1 & S1 & S2 & S2 & S2 & S3 & S3 & S3 \\
Norte & N1 & N2 & N3 & N1 & N2 & N3 & N1 & N2 & N3 \\
$\begin{array}{l}\text { Extensão (km) } \\
\text { No . máx. estações }\end{array}$ & 302 & 297 & 302 & 298 & 293 & 299 & 298 & 293 & 298 \\
intermediárias & 17 & 19 & 18 & 15 & 17 & 16 & 15 & 17 & 16 \\
\hline
\end{tabular}

entre Lisboa e Porto (os voos demoram em média 55 minutos). Estes dois serviços dificilmente poderão subsistir após a introdução da linha de alta velocidade.

O projeto inicial para o corredor Lisboa-Porto sofreu nos últimos anos diversas alterações, estando o traçado final ainda por aprovar. Os vários corredores em estudo são descritos num estudo (ATKEARNEY, 2003) encomendado pela empresa responsável pela implementação da alta velocidade em Portugal, a RAVE - Rede Ferroviária de Alta Velocidade S.A. - e no trabalho de Anciães (2005). Um outro estudo realizado para a RAVE, SDG (2007), revela existirem duas hipóteses para a localização da estação na área de Aveiro: o centro de Aveiro ou Albergaria-a-Velha, um município $15 \mathrm{~km}$ a nascente de Aveiro. O último documento disponibilizado pela RAVE aponta para a construção de uma linha entre Lisboa e Porto com a extensão de $292 \mathrm{~km}$ e com estações intermédias na Região do Oeste (entre Lisboa e Leiria), Leiria, Coimbra e Aveiro/Albergaria-a-Velha (RAVE, 2011).

Com o intuito de estudar os diversos corredores em estudo considerámos três corredores alternativos a norte de Coimbra (N1, N2 e N3) e outros três a sul de Coimbra (S1, S2 e S3), os quais estão representados na Figura 1. No total são considerados 32 munícipios candidatos à localização de estações, cujos nome e abreviatura constam da Tabela 1. A combinação dos corredores norte e sul dá origem a 9 corredores possíveis entre Lisboa e Porto. A Tabela 2 descreve a configuração de cada corredor e respectiva extensão e ainda o número máximo de estações intermédias possíveis. A comparação dos 9 corredores em estudo é feita com base em três indicadores: diminuição dos custos de viagem, demanda captada e receitas de bilheteira.

\section{DADOS DO PROBLEMA}

São necessários essencialmente dois tipos de dados para aplicar o modelo de localização de estações: dados sobre os custos de viagem e dados sobre a demanda potencial da linha de alta velocidade. O cálculo dos custos de viagem e respectiva diminuição foi feito com base na informação que se segue:

- Os custos de viagem na rede rodoviária incluem três componentes: operação dos veículos, valor do tempo e tarifas de pedágio.

- O custo de operação dos veículos foi estimado em 16,478 Euros por $100 \mathrm{~km}$ por veículo. O valor foi obtido usando a metodologia HDM-4 (World Bank, 2010) e inclui consumo de combustível, desgaste de pneus, e manutenção e depreciação do veículo.

- O valor do tempo foi estimado em 12 Euros por hora, em linha com os estudos de transportes recentes feitos em Portugal onde se utilizaram valores de tempo entre 10 e 15 Euros por hora. A este respeito, importa notar que o valor do tempo varia com o modo e motivo de viagem e até mesmo não linearmente dentro do mesmo modo e motivo (ver por exemplo Louviere et al., 2000, e Senna, 1998) e que seria possível atribuir valores de tempo diferentes a modos diferentes sem alteração do modelo.

- As tarifas de pedágio foram consideradas de acordo com os valores reais em vigor.

- A velocidade de circulação na linha de alta velocidade foi estimada em $250 \mathrm{~km} / \mathrm{h}$ (excepto na fase de aceleração e desaceleração).

- O custo dos bilhetes Lisboa-Porto para o serviço de alta velocidade foi estimado em 0.16 Euros $/ \mathrm{km}$, isto é, entre 46,88 e 48,32 Euros dependendo da extensão do corredor. O valor usado no estudo SDG (2009) foi de 49 Euros.

- O tempo perdido em cada transferência intermodal $\left(t^{e}\right)$ foi estimado em 12 minutos (assumção empírica com base em outros estudos de transportes efectuados pelos autores). Notar que é possível atribuir diferentes tempos a cada estação sem alteração do modelo.

- O tempo perdido em cada paragem intermédia $\left(t^{s}\right)$ foi estimado em 9 minutos ( 3 minutos para as fases de aceleração e desaceleração e 6 minutos para o de- 
sembarque e embarque). Este valor é consistente com o estudo SDG (2009) onde a diferença entre uma viagem directa Lisboa-Porto e outra com duas paragens intermédias é de 18 minutos.

A demanda foi calculada utilizando um modelo gravitacional com função de impedância do tipo potência. Este tipo de função apresenta geralmente melhores resultados do que a exponencial para viagens interurbanas (Fotheringham e O'Kelly, 1989). A demanda após a introdução da nova linha de alta velocidade é dada pela seguinte expressão:

$$
q_{i m n j}^{k}=\alpha \frac{w_{i m} w_{n j}}{\left(c_{i m n j}^{k}\right)^{\beta}}
$$

onde $w_{i m}\left(w_{n j}\right)$ é o parâmetro de massa que reflecte o peso do município $i(j)$ como centro gerador de tráfego quando as viagens com origem (destino) nesse município são feitas através de uma estação localizada em $m(n)$, e $\alpha$ e $\beta$ são parâmetros de calibração.

A massa de um município, $i$, para viagens originadas nesse município acedendo à linha de alta velocidade na estação $m$, foi calculada multiplicando o valor da população do município por um factor de redução que reflecte a distância entre o município e a estação e o raio de cobertura da estação, $d_{\max }$, isto é:

$$
w_{i m}=p_{i}\left(1-\frac{d_{i m}}{d_{\text {max }}}\right), i \in \boldsymbol{J}, m \in \boldsymbol{M}: d_{i m} \leq d_{\max }
$$

O cálculo de $w_{n j}$ para viagens destinadas a um município $j$ passando pela estação $n$ é feito com recurso à mesma expressão substituindo os índices $i$ e $m$ pelos índices $n$ e $j$.

Os parâmetros de calibração $\alpha$ e $\beta$ foram estimados respectivamente em 0.35 e 1.20 , utilizando para o efeito a informação de tráfego O/D disponível para a Região Norte de Portugal e técnicas de regressão estatística.

O raio de cobertura das estações $\left(d_{\max }\right)$ foi estimado em $50 \mathrm{~km}$. O mesmo valor foi usado no estudo SDG (2007). Por fim, definiu-se $30 \mathrm{~km}$ como a distância mínima entre estações consecutivas, $l_{\min }$. Este valor foi estabelecido de forma a garantir a não degradação do serviço de alta velocidade até ao nível de um serviço convencional, pois são necessários cerca de $20 \mathrm{~km}$ para acelerar até à velocidade de $250 \mathrm{~km} / \mathrm{h}$ e depois desacelerar até parar.

\section{RESULTADOS}

O modelo de otimização foi aplicado aos nove corredores descritos através de um computador com processador Intel Core 2 Quad Processor Q9550 2.84 GHz e 4 GB de RAM usando o software de otimização FICO Xpress 7.0 (FICO, 2009). Os resultados obtidos com respeito à diminuição dos custos totais de viagem por dia, à demanda captada por dia, às receitas de bilheteira por dia e à localização das estações intermédias estão sintetizados na Tabela 3 , da qual consta também informação sobre o esforço computacional (CPU) necessário para obter as soluções.

Os resultados sintetizados na Tabela 3 permitem comparar os nove corredores analisados. Verifica-se que a solução obtida para o corredor COR8 é aquela que proporciona a maior diminuição de custos de viagem (336,126 Euros por dia) e, simultaneamente, aquela que capta o maior volume de passageiros $(26,334)$ e que apresenta as maiores receitas de bilheteira (858,195 Euros por dia). A solução inclui três estações intermédias, localizadas em Leiria, Coimbra e Oliveira de Azeméis. Comparando esta solução com a solução proposta pela RAVE existem duas diferenças: a estação da Região do Oeste não é seleccionada; e a estação de Aveiro é substituída por uma estação em Oliveira de Azeméis. A solução proposta pela RAVE corresponderá ao corredor COR1. De entre as possibilidades na Região do Oeste para o COR1 a estação que proporciona uma maior diminuição dos custos de viagem (273,961 Euros por dia), ainda assim é a de Torres Vedras com a estação da Região do Oeste que proporciona a melhor solução é a de Torres Vedras, ainda assim $18,5 \%$ pior do que a solução obtida para o COR8 e com a agravante de ser construída mais uma estação e do corredor cerca mais extenso.

Notar que as soluções ótimas obtidas para todos os corredores contemplam apenas três estações intermédias, sendo que uma delas é sempre Coimbra e a Região do Oeste nunca é seleccionada. A construção (ou não) de uma estação em Leiria foi classificada nos estudos iniciais da RAVE como essencialmente política (ver Anciães, 2005). Contudo, neste estudo e exceto nos corredores onde Leiria não é opção (COR4, COR5, e COR6), Leiria foi sempre seleccionada. Já Aveiro, que é incluída em todos os estudos da RAVE (incluindo SDG, 2007 e 2009), nunca foi seleccionada neste estudo, sendo substituída por Oliveira de Azeméis ou Ovar. A justificação prende-se com o facto das áreas de Oliveira de Azeméis e Ovar serem mais populosas do que a área de Aveiro, atraindo mais passageiros.

Tabela 3. Resultados da aplicação do modelo à linha Lisboa-Porto

\begin{tabular}{lllllr}
\hline & $\begin{array}{l}\text { Diminuição dos } \\
\text { custos de viagem } \\
\text { (Euros/dia) }\end{array}$ & $\begin{array}{l}\text { Procura captada } \\
\text { (pax/dia) }\end{array}$ & $\begin{array}{l}\text { Receitas de } \\
\text { bilheteria } \\
\text { (Euros/dia) }\end{array}$ & $\begin{array}{l}\text { Localização } \\
\text { das estações } \\
\text { intermediárias }\end{array}$ & $\begin{array}{l}\text { CPU } \\
\text { (segundos) }\end{array}$ \\
\hline 1 & 291063 & 24974 & 845827 & LEI-CBR-OVA & 762 \\
2 & 316069 & 25860 & 855938 & LEI-CBR-OLA & 3855 \\
3 & 287043 & 24900 & 845688 & LEI-CBR-OVA & 809 \\
4 & 301673 & 22946 & 793832 & TON-CBR-OVA & 1334 \\
5 & 325455 & 23794 & 804016 & TON-CBR-OLA & 1472 \\
6 & 297849 & 22874 & 793414 & TON-CBR-OVA & 1077 \\
7 & 310797 & 25432 & 847883 & LEI-CBR-OVA & 8054 \\
8 & 336126 & 26334 & 858195 & LEI-CBR-OLA & 2128 \\
9 & 306750 & 25352 & 847486 & LEI-CBR-OVA & 807 \\
\hline
\end{tabular}




\section{ANÁLISE DE SENSIBILIDADE}

Por forma a aprofundar o estudo da linha de alta velocidade Lisboa-Porto foi efectuada uma análise de sensibilidade ao preço do bilhete e à decisão crítica de construir ou não uma estação em Aveiro.

\subsection{Preço do bilhete}

O preço pago pelo serviço de alta velocidade representa uma fracção relevante do custo da viagem, podendo, pois, pequenas variações nesse custo ter implicações importantes na atratividade do serviço de alta velocidade.

A influência do preço dos bilhetes no número de estações localizadas, na diminuição dos custos de viagem e na receita de bilheteira foi avaliada através da aplicação do modelo de localização de estações ao COR8 (aquele que apresenta melhores resultados para os dados de base) considerando uma gama de preços de bilhete entre 0,06 e 0,26 Euros por Km. Os resultados obtidos estão sintetizados na Figura 2.

A diminuição do custo dos bilhetes implica o aumento da diminuição dos custos totais de viagem da rede e o aumento do número de estações intermédias localizadas, até ao valor máximo 6 quando o preço do bilhete é 0,06 Euros (mesmo que o preço do bilhete fosse nulo o número ótimo de estações não seria maior). As maiores receitas de bilheteira são obtidas quando o preço dos bilhetes é 0,12 e 0,16 Euros por quilómetro (respectivamente 861180 e 858195 Euros por dia). No entanto as variações são mínimas para a gama de valores de preço do bilhete entre 0,11 e 0,18 . Uma vez que o número de estações localizadas é maior para 0,12 do que para 0,16 (implica um investimento maior), este último será o mais favorável do ponto de vista do operador. Na verdade, 0,16 Euros por quilómetro é um valor muito próximo do valor considerado nos últimos estudos da RAVE.

\subsection{Localização da estação na área de Aveiro}

Seja no centro de Aveiro ou na cidade vizinha de Albergaria-a-Velha todos os estudos encomendados pela RAVE localizam uma estação da futura linha de alta velocidade nesta área. Os resultados obtidos neste estudo permitem-nos concluir que essa não a opção mais vantajosa. De forma a quantificar os prejuízos de localizar uma estação na área de Aveiro aplicámos de novo o modelo aos nove corredores, impondo a localização de uma estação em Aveiro (COR1, COR3, COR4, COR6, COR7, e COR9) ou em Albergariaa-Velha (COR2, COR5, e COR8). Os resultados estão sintetizados na Tabela 4.

Os resultados mostram que a localização de uma estação na área de Aveiro implica um aumento dos custos de viagem entre 2,01 e 3,10\% em relação ao caso de essa localização não ser imposta. As melhores soluções com uma estação localizada em Aveiro e Albergaria-a-Velha são respectivamente obtidas para os corredores COR7 e COR8. Se comparados com a melhor solução do estudo (COR8 sem imposição de localizações), estas soluções representam perdas diárias de 31855 e 9575 Euros no que respeita aos custos de viagem e 3875 e 1779 Euros em termos de receitas de bilheteira. Conclui-se ainda que de entre as duas localizações possíveis na área de Aveiro, Albergaria-a-Velha é mais vantajosa.

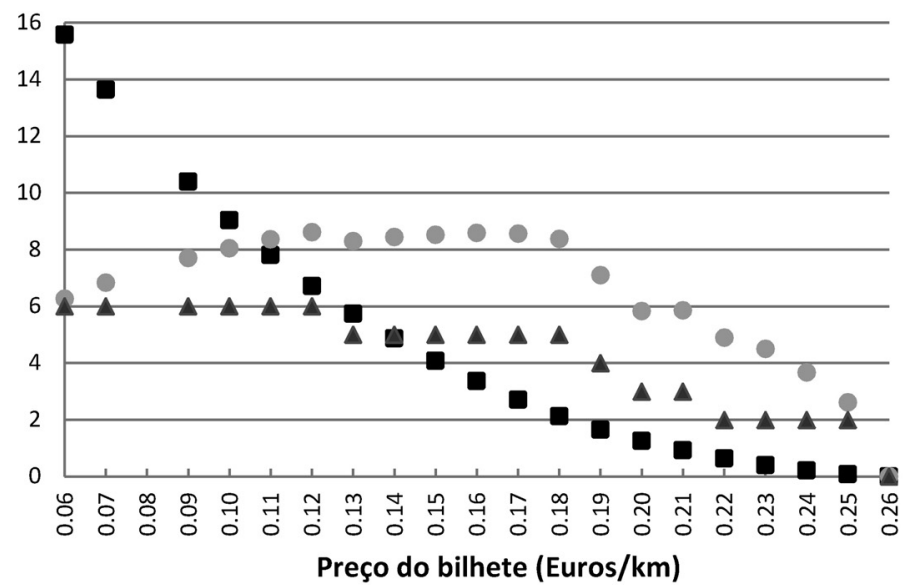

Diminuição dos custos de viagem (x100,000 euros)

- Receita de bilheteira (x100,000 euros)

$\Delta$ Número de estações

Figura 2. Relação entre a diminuição dos custos totais de viagem, o preço dos bilhetes e número de estações localizadas

Tabela 4. Resultados do modelo quando se impõe uma estação na área de Aveiro

\begin{tabular}{llllll}
\hline & $\begin{array}{l}\text { Diminuição dos } \\
\text { custos de viagem } \\
\text { (Euros/dia) }\end{array}$ & $\begin{array}{l}\text { Perda percentual } \\
\text { da diminuição de } \\
\text { custos de viagem }\end{array}$ & $\begin{array}{l}\text { Receitas de } \\
\text { bilheteria } \\
\text { (Euros/dia) }\end{array}$ & $\begin{array}{l}\text { Perda percentual } \\
\text { dos lucros de } \\
\text { bilheteria }\end{array}$ & $\begin{array}{l}\text { Localização } \\
\text { das estações } \\
\text { intermediárias }\end{array}$ \\
\hline 1 & 284794 & 2,15 & 832131 & 1,62 & LEI-CBR-AVE \\
2 & 306278 & 3,10 & 843928 & 1,40 & LEI-CBR-AAV \\
3 & 280724 & 2,20 & 831790 & 1,64 & LEI-CBR-AVE \\
4 & 295608 & 2,01 & 780976 & 1,62 & TON-CBR-AVE \\
5 & 316544 & 2,74 & 793256 & 1,34 & TON-CBR-AAV \\
6 & 291863 & 2,01 & 780683 & 1,60 & TON-CBR-AVE \\
7 & 304271 & 2,10 & 833976 & 1,64 & LEI-CBR-AVE \\
8 & 326551 & 2,85 & 847077 & 1,30 & LEI-CBR-AAV \\
9 & 300315 & 2,10 & 833594 & 1,64 & LEI-CBR-AVE \\
\hline
\end{tabular}




\section{CONCLUSÃO}

Este artigo apresenta um novo modelo de localização de estações ferroviárias e os resultados da sua aplicação à futura linha Lisboa-Porto, uma componente importante do eixo de alta velocidade do Sudoeste Europeu. O objetivo do modelo é maximizar a diminuição dos custos totais de viagem promovidos pela introdução de uma nova linha de alta velocidade. O modelo combina uma série de aspetos até agora nunca abordados simultaneamente. Em particular, considera: o impacto sobre a demanda do tempo perdido em paragens intermédias, a competição de meios de transporte alternativos, e a geração de demanda adicional devido à diminuição dos custos de viagem. Outros aspetos como a velocidade de acesso às estações, as características dinâmicas dos trens, o tempo de paragem dos trens nas estações e o tempo de transferência intermodal, são também contemplados. A aplicação à futura linha Lisboa-Porto ilustrou bem o tipo de resultados que se podem obter através do modelo, e permitiu uma análise aprofundada não só do problema de localização das estações bem como do melhor traçado para a futura linha. Os resultados evidenciam que a solução apresentada nos documentos mais recentes da RAVE não é a mais vantajosa.

O modelo apresentado é de natureza estratégica. No entanto, Bussieck et al. (1997) referem que alguns aspetos de ordem tática devem também ser tidos em consideração aquando da tomada de decisões estratégicas de investimento ferroviário. Em particular, o modelo deveria incluir o planeamento da frota de trens e do serviço prestado (nomeadamente uma tabela horária). Como trabalho futuro, os autores pretendem desenvolver um modelo que aborde simultaneamente os aspetos estratégicos incluídos no modelo e os aspetos táticos mencionados.

\section{AGRADECIMENTOS}

Hugo Repolho agradece o apoio da Fundação para a Ciência e a Tecnologia (bolsa SFRH/BD/28781/2006).

\section{REFERÊNCIAS BIBLIOGRÁFICAS}

Anciães, P. (2005) A influência dos interesses do decisor no processo de escolha pública: Análise geográfica para o caso da parte sul da futura linha de TGV Lisboa-Porto. In: A.R. da Silva; L.L. Souza e J.G. Mendes, (eds.) Planejamento Urbano, Regional, Integrado e Sustentável: Desenvolvimentos Recentes em Portugal e no Brasil, Escola de Engenharia de São Carlos, Universidade de São Paulo, Brazil, 19-38.

ANTT (2011) TAV Brasil. Agência Nacional de Transportes Terrestres. Disponível em: <http://www.tavbrasil.gov.br> (Acesso em 28/02/2011).

ATKEARNEY (2003) Visão Estratégica para a Rede Ferroviária de Alta Velocidade em Portugal: Caracterização dos Corredores Analisados. Lisboa, Portugal.

Bussieck, M. R.; T. Winter e U. T. Zimmermann (1997) Discrete optimization in public rail transport. Mathematical Programming, v. 79, p. 415-444. DOI:10.1007/BF02614327.

Campos, J. e G. De Rus (2009) Some stylized facts about high-speed rail: A review of HSR experiences around the world. Transport Policy, v. 16, n. 1, p. 19-28. DOI:10.1016/j.tranpol.2009.02.008.

De Rus, G. e G. Nombela (2007) Is investment in high speed rail socially profitable? Journal of Transport Economics and Policy, v. 41, n. 1, p. 3-23.

European Commission (2001) European Transport Policy for 2010: Time to Decide. Office for Official Publications of the European Communities, Luxembourg.

European Commission (2005) Trans-European Transport Network: TEN-T Priority Axes and Projects 2005. Office for Official Publications of the European Communities, Luxembourg.
European Union (1996) Decision No. 1692/96/CE of the European Parliament and of the Council of 23 July 1996 on the Community guidelines for the development of the trans-European transport networks. Official Journal of the European Communities 39, L228, 9 September 1996, 1-104.

FICO - Fair Isaac Corporation (2009) Getting Started with Xpress - Release 7. Warwickshire, UK.

Fotheringham, A.S. e M.E. O'Kelly. (1989) Spatial Interaction Models: Formulations and Applications. Kluwer, London, UK. ISSN:0792300211.

Ghoseiri, K.; F. Szidarovszky e M.A. Asgharpour (2004) A multi-objective train scheduling model and solution. Transportation Research Part B, v. 38, n. 10, p. 927-952. DOI:10.1016/j.trb.2004.02.004.

Gross, D.; H.W. Hamacher; S. Horn e A. Schöbel (2009) Stop location design in public transportation networks: Covering and accessibility objectives. TOP, v. 17, n. 2, p. 335-346. DOI:10.1007/s11750008-0061-4.

Hamacher, H.W.; A. Liebers; A. Schöbel; D. Wagner e F. Wagner (2001) Locating new stops in a railway network. Electronic Notes in Theoretical Computer Science, v. 50, n. 1, p. 13-23. DOI: 10.1016/S1571-0661(04)00162-8.

Kranakis, E.; P. Penna; K. Schlude; D. Taylor e P. Widmayer (2003) Improving customer proximity to railway stations. Lecture Notes in Computer Science, v. 2653, p. 264-276. DOI:10.1007/3-54044849-7 30.

Laporte, G.; J.A. Mesa e F.A. Ortega (1998) Locating stations on rapid transit lines. Centre for Research on Transportation (Publication CRT-98-22), Montreal, Canada.

Laporte, G.; J. A. Mesa e F. A. Ortega (2000) Optimization methods for the planning of rapid transit systems. European Journal of Operational Research, v. 122, n. 1, p. 1-10. DOI:10.1016/S03772217(99)00016-8.

Marin, Á.G. e P. Jaramillo (2009) Urban rapid transit network design: Accelerated benders decomposition. Annals of Operations Research 169(1), 35-53.DOI:10.1007/s10479-008-0388-0.

Nash, C. (2009) When to invest in high-speed rail links and networks? OECD Publishing, OECD/ITF Joint Transport Research Centre Discussion Papers 2009-16, Paris, France. DOI:10.1787/ $5 \mathrm{kmmr} 3 \mathrm{gg} 21 \mathrm{hk}$-en

Ortúzar, J.D. e L. G. Willumsen (2001) Modelling Transport. Wiley, Chichester, UK.

RAVE (2011) The Portuguese High-Speed Railway Project. Lisbon, Portugal.

Schöbel, A. (2005) Locating stops along bus or railway lines: A bicriteria problem. Annals of Operations Research, v. 136, n. 1, p. 211227. DOI:10.1007/s10479-005-2046-0.

Schöbel, A., H. Hamacher, A. Liebers, D. Wagner (2002) The continuous stop location problem in public transportation networks. Technical Report, v. 81, Wirtschaftsmathematik, University of Kaiserslautern, Germany.

SDG (2007) Modelo Integrado de Procura de Passageiros - Relatório 2: Estimativas de Procura de Passageiros e Receitas. Steer Davies Gleave. Londres, Reino Unido.

SDG (2009) Análise Custo-Benefício da Ligação de Alta Velocidade Ferroviária entre Lisboa e Porto - Relatório Final. Steer Davies Gleave. Londres, Reino Unido.

TIS.pt (2007) Análise Custo-Beneficio das Linhas de Alta Velocidade Lisboa-Porto e Lisboa-Madrid-Relatório Final. Lisboa, Portugal.

Vuchic, V.R. (1969) Rapid transit interstation spacing for maximum number of passengers. Transportation Science, v. 3, n. 4, p. 214-232. DOI:10.1287/trsc.3.3.214.

Vuchic, V. R. e G. F. Newell (1968) Rapid transit interstation spacing for minimum travel time. Transportation Science, v. 2, n. 4, p. 303339. DOI: $10.1287 /$ trsc.2.4.303.

Vuchic, V. R. e J. M. Casello (2002) An evaluation of maglev technology and its comparison with high speed rail. Transportation Quarterly, v. 56, n. 2, p. 33-49.

World Bank (2010) HDM-4 Road Use Costs Model Version 2.00 Documentation. Washington DC, USA. 